\title{
Dynamics of seeded blobs under the influence of inelastic neutral interactions
}

Thrysøe, A.S.; Naulin, V.; Nielsen, A. H.; Juul Rasmussen, J.

Published in:

Physics of Plasmas

Link to article, DOI:

$10.1063 / 5.0003262$

Publication date:

2020

Document Version

Publisher's PDF, also known as Version of record

Link back to DTU Orbit

Citation (APA):

Thrysøe, A. S., Naulin, V., Nielsen, A. H., \& Juul Rasmussen, J. (2020). Dynamics of seeded blobs under the influence of inelastic neutral interactions. Physics of Plasmas, 27(5), [052302].

https://doi.org/10.1063/5.0003262

\section{General rights}

Copyright and moral rights for the publications made accessible in the public portal are retained by the authors and/or other copyright owners and it is a condition of accessing publications that users recognise and abide by the legal requirements associated with these rights.

- Users may download and print one copy of any publication from the public portal for the purpose of private study or research.

- You may not further distribute the material or use it for any profit-making activity or commercial gain

- You may freely distribute the URL identifying the publication in the public portal

If you believe that this document breaches copyright please contact us providing details, and we will remove access to the work immediately and investigate your claim. 


\section{Dynamics of seeded blobs under the influence of inelastic neutral interactions}

Cite as: Phys. Plasmas 27, 052302 (2020); https://doi.org/10.1063/5.0003262

Submitted: 03 February 2020 . Accepted: 18 April 2020 . Published Online: 12 May 2020

A. S. Thrys $\varnothing$ e (D), V. Naulin (D), A. H. Nielsen (D), and J. Juul Rasmussen (D)

\section{COLLECTIONS}

F This paper was selected as Featured
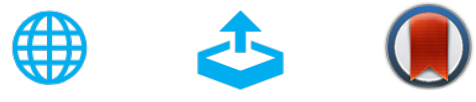

\section{ARTICLES YOU MAY BE INTERESTED IN}

How blobs affect plasma dynamics in tokamaks

Scilight 2020, 201103 (2020); https://doi.org/10.1063/10.0001302

MHD-blob correlations in NSTX

Physics of Plasmas 27, 052505 (2020); https://doi.org/10.1063/5.0006515

A new explanation of the sawtooth phenomena in tokamaks

Physics of Plasmas 27, 032509 (2020); https://doi.org/10.1063/1.5140968

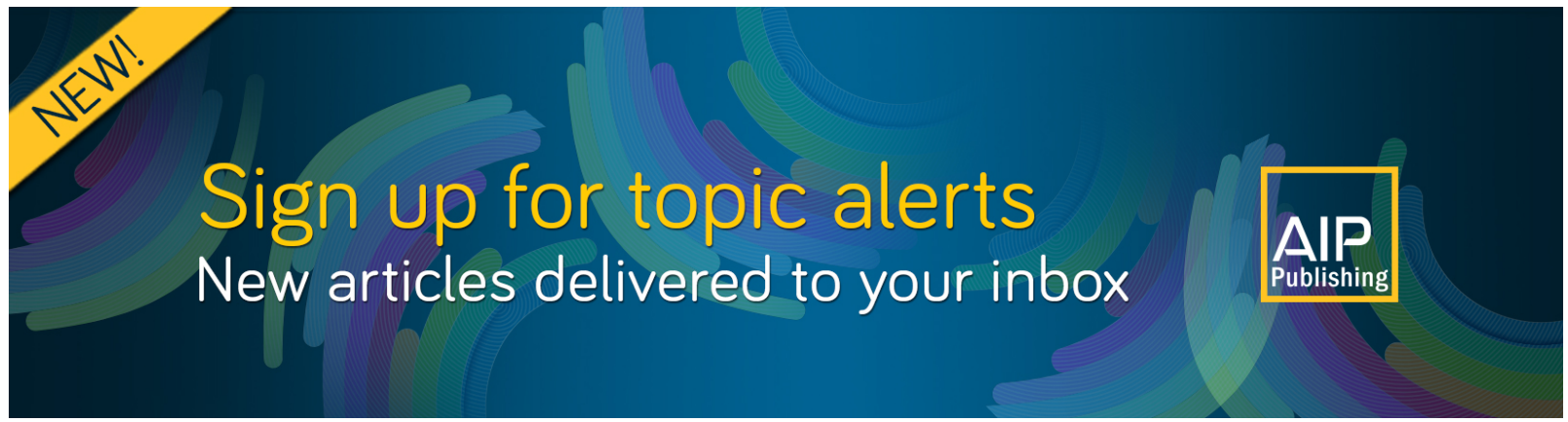




\title{
Dynamics of seeded blobs under the influence of inelastic neutral interactions
}

\author{
Cite as: Phys. Plasmas 27, 052302 (2020); doi: 10.1063/5.0003262 \\ Submitted: 3 February 2020 - Accepted: 18 April 2020 . \\ Published Online: 12 May 2020
}

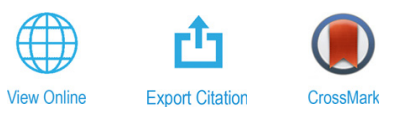

\section{A. S. Thrysøe, ${ }^{a)}$ (D) V. Naulin, (D) A. H. Nielsen, (D) and J. Juul Rasmussen (D)}

\section{AFFILIATIONS}

PPFE, Department of Physics, DTU, 2800 Kgs. Lyngby, Denmark

a) Author to whom correspondence should be addressed: alec@fysik.dtu.dk

\begin{abstract}
Field-aligned filaments, the so-called blobs, born at the edge of the magnetically confined region of tokamaks propagate radially outward into the scrape-off layer (SOL) region that allows for a substantial population of neutral particles compared to the region of confinement. The electrons and ions constituting the blob undergo both elastic and inelastic collisions with the neutral particles, and the latter leads to sources and sinks of the blob density, momentum, and heat. The influence of the inelastic collisions with neutrals on the evolution of seeded blobs is investigated numerically by the nHESEL drift-fluid model through a series of discrete scans in interactions, active source terms, and blob plasma parameters. In light of the results, the potential influence of local inelastic collisions on the SOL density shoulder formation is discussed. It is found that density sources increase the blob compactness, which delays the blob dispersion and decreases the dispersion rate. Density sources or momentum sinks also influence the blob dynamics by increasing the vorticity layer around the perturbation, whereas the pressure sources/sinks only affect the blob dynamics marginally. The change to the vorticity structure leads, in most cases, to a decrease in the radial velocity of the blob center of mass, although, at high source rates, a radial acceleration of the blob center of mass is also observed. Density sources may, thus, contribute to shoulder formation not only by increasing the density locally but also by changing the filament dynamics.
\end{abstract}

Published under license by AIP Publishing. https://doi.org/10.1063/5.0003262

\section{INTRODUCTION}

Cross-field transport in the edge and scrape-off layer (SOL) regions in toroidal magnetically confined plasmas is highly intermittent. Particles and energy are transported across the magnetic field lines in coherent field-aligned filaments, ${ }^{1,2}$ often denoted blobs, which are present for all machine sizes. ${ }^{3}$ Blobs typically have a width of a few centimeters perpendicular to the magnetic field lines, but extend tens of meters in the direction parallel to the field lines. The strong anisotropy of blobs allows for, to some extent, treating the parallel and cross-field dynamics separately.

The underlying mechanism for cross-field blob transport is well understood. ${ }^{4,5}$ Diamagnetic and curvature drifts polarize the blob in the direction perpendicular to both the magnetic field and its curvature/gradient. The polarization gives rise to a radially outward $\mathbf{E} \times \mathbf{B}$ drift of the filament. The cross-field filamentary transport is often studied numerically in an isolated manner by simulating the so-called seeded blobs. The seeded blob is a Gaussian density and temperature perturbation on top of a flat background in a two-dimensional driftplane domain. The blobs are typically studied in the so-called inertial regime, where the parallel current is disregarded. In the case of cold ions (i.e., $T_{\mathrm{i}}=0$ ), the blob evolves into a mushroom shape that characterizes interchange-driven structures. Finite temperature ion dynamics, however, break the symmetry in the $\mathbf{B} \times \nabla \mathbf{B}$ direction $^{6,7}$ and cause the blob to remain more compact, as seen for the two seeded blobs (both with finite ion temperatures) in Fig. 1.

Together with the SOL geometry and plasma edge conditions, the neutrals are the main players in defining the plasma properties in the edge and SOL regions. A topic of particular current interest is that of the formation of a density shoulder in the SOL region. The density shoulder usually forms in high density regimes, which can be obtained by intense neutral gas puff fueling. There are several possible underlying mechanisms in play for the cause of the shoulder formation. ${ }^{8,9}$ Among the candidates are enhanced filamentary transport due to detachment caused by increased collisionality in the divertor region and increased ionization in the SOL region due to a higher neutral density.

The addition of a neutral background to the seeded blob studies has been studied previously, where inertial models have been extended to also include the effect of friction terms from elastic plasma-neutral collisions. $^{10,11}$ Elastic collisions do not, however, account for, e.g., 

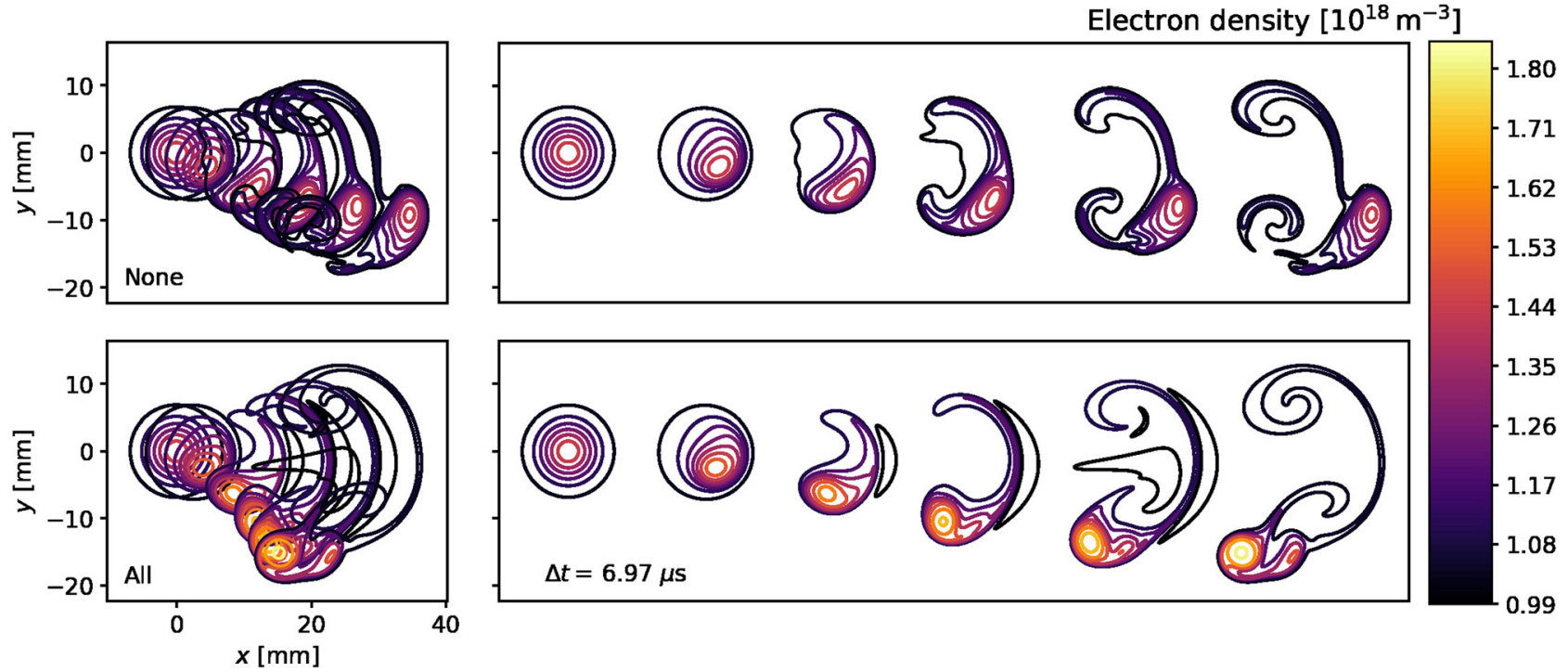

1.35

1.26

1.17

1.08

FIG. 1. Evolution of a seeded blob in a time span of $34.85 \mu \mathrm{s}$. The upper left frame shows the evolution without the influence of neutral interactions, and the lower left frame is with neutral interactions. In the right column of the frames, the snapshots are displaced horizontally for better visualization.

ionization of molecules and atoms or charge-exchange collisions between ions and atoms. The inelastic collisions introduce additional drift terms, ${ }^{12}$ which influence the filamentary transport and may contribute to observed SOL transport phenomena such as shoulder formation.

This paper presents the effects of source terms from the inelastic neutral interactions in the inertial seeded blob scheme. In Sec. II, the applied model is presented, and the initial conditions are defined. The results are presented in Sec. III, in which the effects of the neutral interactions are assessed through a scan in interaction terms and another scan in the source terms. The section also presents scans in the plasma and neutral density parameters. The conclusions drawn from the numerical results are augmented with a more detailed discussion in Sec. IV. Conclusions are summarized in Sec. V.

\section{MODEL}

In this section, the model for studying the effects of the dominant inelastic interactions with neutrals is presented. The model equations are described in detail in Ref. 12 and briefly reviewed in Sec. II A. The particular setup for seeded blob studies is defined in Sec. II B.

\section{A. Model equations}

The evolution of the seeded blobs is described by the nHESEL equations, consisting of the HESEL model for the plasma field evolution $^{13-15}$ coupled to a module for neutral particle fluid transport and interactions. $^{12}$ HESEL is a drift-fluid model derived from the Braginskii equations ${ }^{16}$ and describe the transport of electron density $n$, generalized vorticity $\omega$, and electron and ion pressures $p_{\mathrm{e}, \mathrm{i}}$ in a driftplane perpendicular to the magnetic field lines in a slab located at the outboard midplane of a tokamak. The Bohm normalized nHESEL equations read

$$
\mathrm{d}_{t} n+n \mathcal{C}(\phi)-\mathcal{C}\left(p_{\mathrm{e}}\right)=\Lambda^{n}+S^{n},
$$

$$
\begin{gathered}
\mathrm{d}_{t}^{0} \omega+\left\{\nabla \phi, \nabla p_{\mathrm{i}}\right\}-\mathcal{C}\left(p_{\mathrm{e}}+p_{\mathrm{i}}\right)=\Lambda^{\omega}+S^{\omega}, \\
\frac{3}{2} \mathrm{~d}_{t} p_{\mathrm{e}}+\frac{5}{2} p_{\mathrm{e}} \mathcal{C}(\phi)-\frac{5}{2} \mathcal{C}\left(p_{\mathrm{e}}^{2} / n\right)=\Lambda_{\mathrm{e}}^{p}+S_{\mathrm{e}}^{p}, \\
\frac{3}{2} \mathrm{~d}_{t} p_{\mathrm{i}}+\frac{5}{2} p_{\mathrm{i}} \mathcal{C}(\phi)+\frac{5}{2} \mathcal{C}\left(p_{\mathrm{i}}^{2} / n\right)-p_{\mathrm{i}} \mathcal{C}\left(p_{\mathrm{e}}+p_{\mathrm{i}}\right)=\Lambda_{\mathrm{i}}^{p}+S_{\mathrm{i}}^{p} .
\end{gathered}
$$

Here, $\omega=\nabla_{\perp}^{2} \phi+\nabla_{\perp}^{2} p_{\mathrm{i}}$ is the generalized vorticity, $\phi$ is the electrostatic potential, and $\mathrm{d}_{t}=\partial_{t}+B^{-1}\{\phi, \quad\}$ is the convective derivative with $\mathbf{E} \times \mathbf{B}$ advection written in terms of the Poisson bracket $\{\phi, f\}=\partial_{x} \phi \partial_{y} f-\partial_{x} f \partial_{y} \phi$. The curvature operator is defined as $\mathcal{C}(f)=-\frac{\rho_{\mathrm{s}}}{R} \partial_{y} f$, where $R$ is the major radius and $\rho_{\mathrm{s}}=\sqrt{\frac{\frac{T}{\mathrm{e} 0}_{m_{\mathrm{i}} \Omega_{\mathrm{c}}}}{{ }^{2}}}$ is the cold-ion hybrid thermal gyro-radius, which is given by the reference electron temperature $T_{\mathrm{e} 0}$, the ion mass $m_{\mathrm{i}}$, and the ion cyclotron frequency $\Omega_{\mathrm{ci}}$. The $\Lambda_{-, \mathrm{e}, \mathrm{i}}^{n, \omega, p}$ terms contain terms resulting from the parameterization of collisions and parallel transport and are specified in Ref. 15. In the inertial blob studies for this paper, the terms arising from the parameterization of parallel dynamics are truncated.

The source terms $S_{-, e, i}^{n, \omega, p}$ on the RHS of Eqs. (1)-(4) are provided by the neutral particle module and describe sources from inelastic collisions between the charged particles and neutral atoms and molecules. The model only considers neutrals of the same atomic number as the ions so that in, e.g., a deuterium plasma, only interactions with neutral $\mathrm{D}$ and $\mathrm{D}_{2}$ particles are considered. The interactions included in the model are dissociation of molecules (5), ionization and dissociation of molecules (6), ionization of atoms (7), and charge-exchange collisions between atoms and ions (8):

$$
\text { Dissociation } \mathrm{D}_{2}+\mathrm{e} \rightarrow 2 \mathrm{D}+\mathrm{e},
$$

Molecular ionization $\quad \mathrm{D}_{2}+\mathrm{e} \rightarrow \mathrm{D}_{2}^{+}+2 \mathrm{e} \rightarrow \mathrm{D}+\mathrm{D}^{+}+2 \mathrm{e}$,

$$
\text { Atomic ionization } \mathrm{D}+\mathrm{e} \rightarrow \mathrm{D}^{+}+2 \mathrm{e},
$$

Charge-exchange $\mathrm{D}+\mathrm{D}^{+} \rightarrow \mathrm{D}^{+}+\mathrm{D}$. 
In the full nHESEL model, the transport of neutrals is described by diffusion, and the inelastic interactions are sources and sinks for the neutral densities. Those features, however, are disabled for the current study and the neutrals are described as a fixed background through which the seeded blob propagates. The source terms to the plasma fields from interactions with the neutrals are

$$
\begin{gathered}
S^{n}=S_{\mathrm{m} . \mathrm{iz}}^{n}+S_{\mathrm{a} . \mathrm{iz}}^{n}, \\
S^{\omega}=-\nabla \cdot\left(n \boldsymbol{u}_{\mathrm{i}}^{S}\right), \\
S_{\mathrm{e}}^{p}=S_{\mathrm{e}, \mathrm{dis}}^{p}+S_{\mathrm{e}, \mathrm{m} . \mathrm{iz}}^{p}+S_{\mathrm{e}, \mathrm{a} . \mathrm{iz}}^{p}, \\
S_{\mathrm{i}}^{p}=S_{\mathrm{i}, \mathrm{m} . \mathrm{iz}}^{p}+S_{\mathrm{i}, \mathrm{a} . \mathrm{iz}}^{p}+S_{\mathrm{i}, \mathrm{cx}}^{p}-\frac{3}{2} \nabla \cdot\left(p_{\mathrm{i}} \boldsymbol{u}_{\mathrm{i}}^{\mathrm{S}}\right)-p_{\mathrm{i}} \nabla \cdot \boldsymbol{u}_{\mathrm{i}}^{\mathrm{S}} .
\end{gathered}
$$

Here, the ion source drift is

$$
\boldsymbol{u}_{\mathrm{i}}^{\mathrm{S}}=\frac{\boldsymbol{R}_{\perp \text { in }}^{\mathrm{cx}}-\boldsymbol{R}_{\perp \mathrm{ni}}^{\mathrm{cx}}}{n_{\mathrm{i}} \Omega_{\mathrm{ci}}}-\frac{S^{n}+S^{\mathrm{cx}}}{n_{\mathrm{i}} \Omega_{\mathrm{ci}}} \boldsymbol{u}_{\mathrm{i}},
$$

and the normalized interaction specific source terms are

$$
\begin{gathered}
S_{\mathrm{m} . \mathrm{iz}}^{n}=n n_{\mathrm{m}}\left\langle\sigma_{\mathrm{m} . \mathrm{iz}} v\right\rangle, \\
S_{\mathrm{a} . \mathrm{iz}}^{n}=n n_{\mathrm{a}}\left\langle\sigma_{\mathrm{a} . \mathrm{iz}} v\right\rangle, \\
S^{\mathrm{cx}}=\frac{1}{2} n n_{\mathrm{a}}\left\langle\sigma_{\mathrm{cx}} v\right\rangle, \\
S_{\mathrm{e}, \mathrm{dis}}^{p}=-\phi_{\mathrm{dis}} n n_{\mathrm{m}}\left\langle\sigma_{\mathrm{dis}} v\right\rangle, \\
S_{\mathrm{e}, \mathrm{m} . \mathrm{iz}}^{p}=\left[\frac{3}{2} \mu T_{\mathrm{m}}+\frac{1}{2} \mu u_{\mathrm{e}}^{2}-\phi_{\mathrm{m} . \mathrm{iz}}\right] S_{\mathrm{m} . \mathrm{iz}}^{n}, \\
S_{\mathrm{e}, \mathrm{a} . \mathrm{iz}}^{p}=\left[\frac{3}{2} \mu T_{\mathrm{a}}+\frac{1}{2} \mu u_{\mathrm{e}}^{2}-\phi_{\mathrm{a} . \mathrm{iz}}\right] S_{\mathrm{a} . \mathrm{iz}}^{n}, \\
S_{\mathrm{i}, \mathrm{m} . \mathrm{iz}}^{p}=\left[\frac{3}{2} T_{\mathrm{m}}+\frac{1}{2} u_{\mathrm{e}}^{2}\right] S_{\mathrm{m} . \mathrm{iz}}^{n}, \\
S_{\mathrm{i}, \mathrm{a} . \mathrm{iz}}^{p}=\left[\frac{3}{2} T_{\mathrm{a}}+\frac{1}{2} u_{\mathrm{i}}^{2}\right] S_{\mathrm{a} . \mathrm{i} z}^{n}, \\
S_{\mathrm{i}, \mathrm{cx}}^{p}=\frac{1}{2} u_{\mathrm{i}}^{2} S^{\mathrm{cx}}-\boldsymbol{u}_{\mathrm{i}} \cdot \boldsymbol{R}_{\mathrm{in}}^{\mathrm{cx}}+Q_{\mathrm{in}}^{\mathrm{cx}}-Q_{\mathrm{ni}}^{\mathrm{cx}} .
\end{gathered}
$$

Here, $n_{\mathrm{m}, \mathrm{a}}$ are the molecule and atom densities, $\left\langle\sigma_{\rho} v\right\rangle$ is the reaction rate coefficients for the interactions $\rho=\{$ dis, m.iz, a.iz, cx $\}, \phi_{\rho}$ is the ionization/dissociation potential associated with the interactions $\rho$, $\mu$ is the electron to ion mass ration, and the ion to atom mass ratio is assumed to be $1, T_{\mathrm{m}, \mathrm{a}}$ are the molecule and average atom temperatures, $\boldsymbol{u}_{\mathrm{e}}=\boldsymbol{u}_{\mathrm{i}}$ are the leading order plasma velocity from $\mathbf{E} \times \mathbf{B}$ advection, and $\boldsymbol{R}_{\mathrm{ni} \text {,in }}^{\mathrm{cx}}$ and $Q_{\mathrm{ni}, \text { in }}^{\mathrm{cx}}$ are the friction and frictious heating terms due to charge-exchange collisions defined in Ref. 12.

The nHESEL model describes the neutral atoms as two isothermal fluids at distinct ("warm" and "hot") temperatures. The warm neutrals have a temperature of $2 \mathrm{eV}$ to resemble the Franck-Condon neutrals, and the hot neutrals have a temperature similar to the ion edge temperature to resemble warm neutrals that have undergone charge-exchange collisions with ions. In the seeded blob setup applied here, the two neutral atom species at different temperatures each constitute half of the total atom density.

\section{B. Seeded blob setup}

The seeded blob is initialized as a Gaussian perturbation of width $\sigma$ to the plasma density and temperatures on top of a flat background,

$$
f(x, y, 0)=f_{0}\left[1+\frac{1}{2} \exp \left(-\frac{\left(x-x_{0}\right)^{2}+\left(y-y_{0}\right)^{2}}{2 \sigma^{2}}\right)\right],
$$

for $f=n, T_{\mathrm{e}, \mathrm{i}}$. The initial position is defined by the coordinate set $\left(x_{0}, y_{0}\right)$, and the amplitude of the perturbation is half of the value of the background. The generalized vorticity $\omega$ is zero at initialization.

The seeded blob is centered in a square box of side length $40 \sigma$ to ensure that the boundaries are far enough away to not influence the blob propagation. In the $y$-direction, which corresponds to the poloidal direction, the boundary is periodic, and in the $x$-direction, which corresponds to the radial direction, the boundaries have Neumann conditions. The remaining geometric parameters are similar to those for a medium-sized tokamak with magnetic field on-axis $B_{0}=2 \mathrm{~T}$, safety factor $q_{95}=5$, the minor radius $a=0.5 \mathrm{~m}$, and the major radius $R=1.5 \mathrm{~m}$. The ions have mass number $A=2$ and charge number $z=1$ (deuterium). The default reference and initialization parameters are $n_{0}=n_{\mathrm{m}, \mathrm{a}}=1 \times 10^{18} \mathrm{~m}^{-3}, T_{\mathrm{e}, \mathrm{i} 0}=10 \mathrm{eV}, \quad$ and $\sigma=10 \rho_{\mathrm{s}}$. The setup is similar to that of Ref. 6 .

For the seeded blobs, the source terms from neutral interactions in Sec. II A are modified so that the value of the plasma density is only that of the perturbation. This is done by subtracting the background value from $n$ used when calculating the source terms. If this is not done, the background interacts with the neutrals and the effects of the interactions on the blob dynamics are obscured.

The nHESEL seeded blob model equations are solved in the BOUT ++ framework $^{17}$ on a spatial grid of $2048 \times 2048$ grid-points. The spatial length is normalized by $\rho_{s}$, and the time is normalized by the inverse ion cyclotron frequency. All simulations are run for a temporal extent of $2500 \Omega_{\mathrm{ci}}^{-1}=34.85 \mu \mathrm{s}$.

\section{BLOB DYNAMICS}

This section presents the results from the numerical simulations of seeded blobs under the influence of inelastic neutral interactions. In Fig. 1, two blob evolution scenarios are shown. In the upper frames, the blob evolves without the influence of neutral interactions, whereas in the lower frames, the blob is subject to the source terms presented in Sec. II A. The influence of the individual interactions is investigated through a discrete scan in Sec. III A. Section III B substantiates the results of Sec. III A through a discrete scan in the plasma source terms, i.e., $S_{-, \text {, i. }}^{n, \omega, p}$ in (9)-(12). The evolution of the seeded blobs in Secs. III A and III $B$ is measured by the spatial trace of the center-of-mass (CoM) of the perturbation

$$
x_{\mathrm{CoM}}(t)=\frac{\int \mathrm{d} \boldsymbol{x}\left(n(x, y, t)-n_{0}\right) \boldsymbol{x}}{\int \mathrm{d} \boldsymbol{x}\left(n(x, y, t)-n_{0}\right)},
$$

the temporal evolution of the CoM velocity

$$
v_{\mathrm{CoM}}(t)=\partial_{t} x_{\mathrm{CoM}}(t),
$$

the evolution of the so-called compactness ${ }^{6,7}$ 


$$
I_{\mathrm{C}}(t)=\frac{\int \mathrm{d} \boldsymbol{x}\left(n(x, y, t)-n_{0}\right) h(x, y, t)}{\int \mathrm{d} \boldsymbol{x}\left(n(x, y, 0)-n_{0}\right) h(x, y, 0)},
$$

with the Heaviside function defined by

$$
h(x, y, t)=\left\{\begin{array}{lll}
0 & \text { if } \quad & \left(x-x_{\max }(t)\right)^{2}+\left(y-y_{\max }(t)\right)^{2} \geq \sigma^{2}, \\
1 & \text { if } \quad\left(x-x_{\max }(t)\right)^{2}+\left(y-y_{\max }(t)\right)^{2}<\sigma^{2},
\end{array}\right.
$$

and the evolution of the thermal and kinetic blob energies

$$
\begin{gathered}
E_{\sigma, \text { th }}(t)=\frac{3}{2} \int \mathrm{d} x p_{\sigma}(x, y, t), \\
E_{\text {kin }}(t)=\frac{1}{2} \int \mathrm{d} \boldsymbol{x}\left|\nabla_{\perp} \phi(x, y, t)\right|^{2},
\end{gathered}
$$

for the $\sigma=\mathrm{e}$, i electron and ion species. These are all metrics that are usually calculated to describe the motion of seeded blobs. In addition to the original compactness defined in (26), we define the source compensated compactness as

$$
\tilde{I}_{\mathrm{C}}(t) \equiv I_{\mathrm{C}}(t) \frac{\int \mathrm{d} \boldsymbol{x}\left(n(x, y, 0)-n_{0}\right)}{\int \mathrm{d} \boldsymbol{x}\left(n(x, y, t)-n_{0}\right)} .
$$

In the case of vanishing density sources and sinks, the expressions in (26) and (30) are identical, but the source compensated compactness serves the purpose of the compactness better in the presence of a finite density source. The original definition of compactness cannot separate an increase in the overall density from a local accumulation of particles although it is only meant for gauging the latter. For a density source, the compactness could increase even though the blob disperses, which is avoided with the source compensated compactness.

Finally, a scan in $T_{\mathrm{e}, \mathrm{i} 0}, n_{0}, \sigma, n_{\mathrm{m}, \mathrm{a}}$ is carried out to ensure that the plasma parameter values used in the previous scans are representative as well as probing limit cases for the plasma/neutral parameters. The results of the parameter scans are discussed in Sec. III C.

\section{A. Neutral interaction scan}

Figure 1 shows that the dynamics of a seeded blob with and without the influence of inelastic neutral interactions differ. In this section, the four interactions, i.e., dissociation of molecules, ionization of molecules followed by dissociation, ionization of atoms, and chargeexchange between atoms and ions, shown in (5)-(8), are investigated individually. The trajectories of the blob CoMs for the set of interactions are shown in Fig. 2. It is observed that the CoM trajectories for the blob without neutral interactions and that with only dissociation are almost identical. The CoM trajectories for the density source interactions, i.e., ionization of molecules and atoms, and for that which include chargeexchange collisions are bent slightly in the $\mathbf{B} \times \nabla \mathrm{B}$ direction. The CoM trajectory of the blob, for which all interactions are included, diverges significantly from the others, and in a way that appears to be more than the sum of the deviations introduced by the individual interactions.

The slope of the radial position in the lower left frame of Fig. 2, or equivalently the plots in Fig. 3, likewise shows that the CoM velocity is mostly unaffected by the individual interactions, but that the radial velocity drops to a much lower value after reaching the maximum
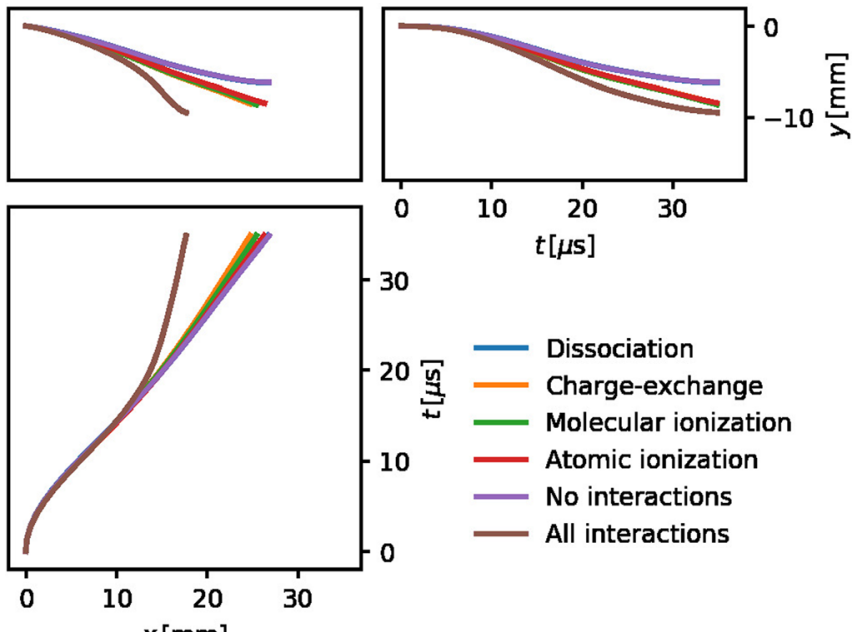

FIG. 2. Trajectory of the seeded blob center of mass position under the influence of different neutral interactions. The $x$ - and $y$-axes are shared in the aligned frames so that the plot in the upper left corner shows the trajectory in space. The trajectories for no interactions and "dissociation" are almost identical, and "molecular ionization," "atomic ionization," and "charge-exchange" form another group of similar dynamics. The blob with all interactions included behaves significantly different from the others.

radial velocity for the case with all interactions included than for those with isolated interactions.

The evolution of the blob compactness metrics shown in Fig. 4 confirms what one could expect from (26): that the interactions that introduce density sources affect the compactness the most. The original compactness (26) increases the compactness to values well above 1 , which has not been observed previously where the simulations have not included inelastic neutral interactions. ${ }^{6,7}$ The source compensated compactness (30) indicates that the blobs still dissipate as for the cases

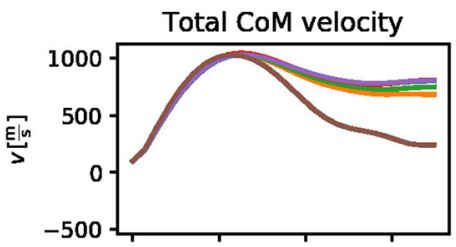

Poloidal CoM velocity
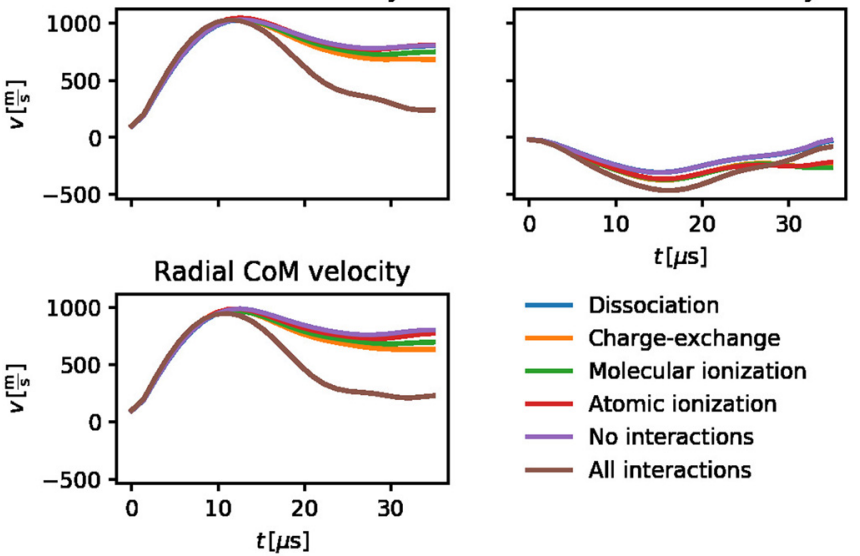

FIG. 3. Evolution of the seeded blob center of mass velocity under the influence of different neutral interactions. The velocity evolves similar for all single interactions, although the same fragmentation as in Fig. 2 is observed for the poloidal velocity component, and for the full velocity for the blob influenced by all interactions. 

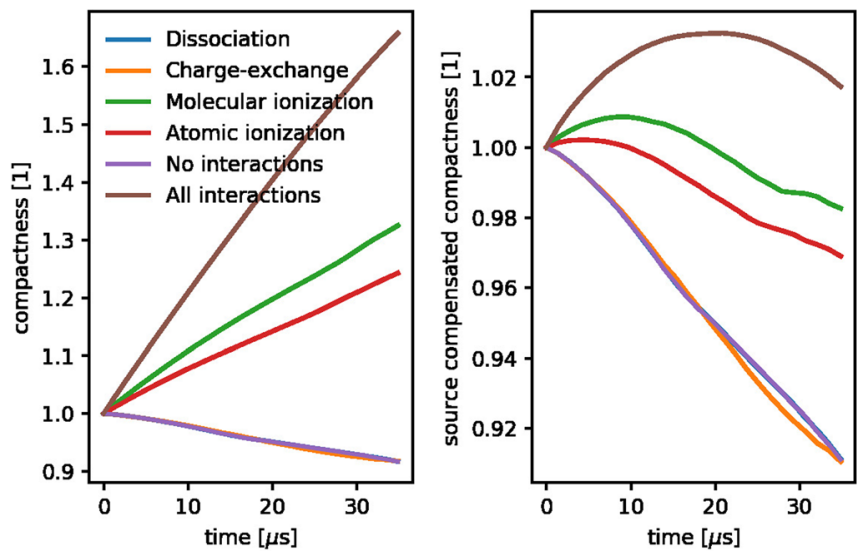

FIG. 4. Compactness and source compensated compactness of the perturbation in the interaction scan. The graphs for the no interactions, dissociation, and chargeexchange scans are almost overlapping, and only the interactions that result in a density source influence the filament compactness significantly.

without a density source, but the dissipation rate is delayed and slowed down by the presence of a density source. The later and slower dissipation of the fueled blobs may have the effect of increasing the lifetime of the blob.

Finally, the difference in the evolution of the thermal energies, shown in Fig. 5, also appears to be driven by the density source interactions. The sink introduced by the ionization potential depletes the electron thermal energy, whereas the creation of new ions increases the ion thermal energy. The ion pressure source from the creation of new particles (20) and (21) (and, in particular, the dependence on the ion flow velocity) may seem peculiar, but it is necessary for the conservation of energy. It results from the (questionable) assumption that
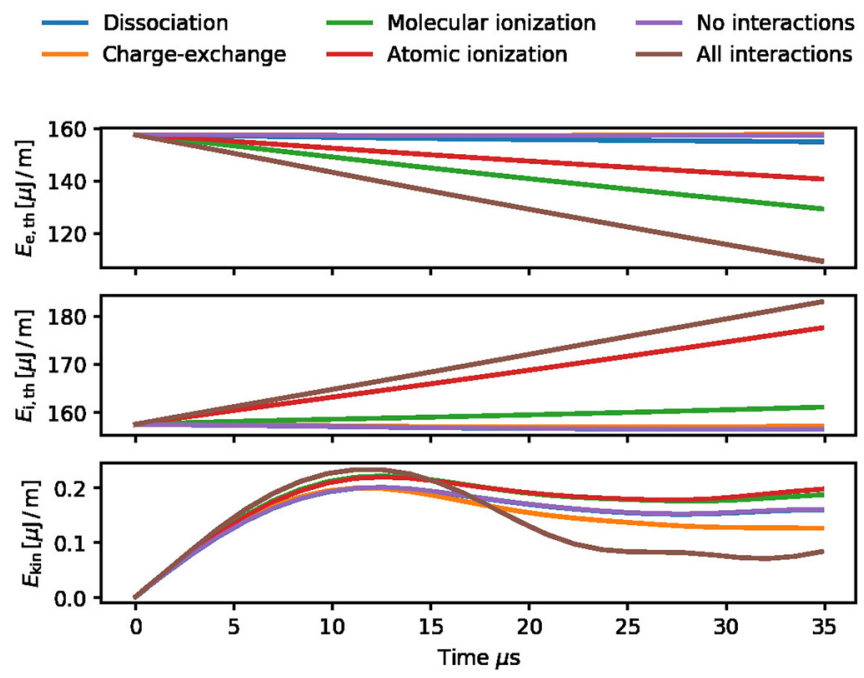

FIG. 5. Evolution of thermal electron (top frame) and ion (middle frame) energies, and the blob kinetic energy (bottom frame), under the influence of neutral interactions. The interactions that give rise to a density source have the largest influence on the energy evolution. the velocity distribution of the ion fluid remains Maxwellian under the addition of particles that originate from a fluid with flow velocity and temperature disparate from those of the ions. A separate publication on this particular matter is under preparation. It is also observed that the kinetic energy is higher for the density sources and lower for charge-exchange, whereas the case with only dissociation, which only introduces an electron pressure sink, is almost indistinguishable from that without neutral interactions. For the case with all interactions included, the kinetic energy appears to follow that of the cases with only density source interactions in the early stage but diverges from this trend after reaching the maximum energy.

In summary, the interactions that introduce the density sources, and in some cases also charge-exchange collisions, seem to influence the seeded blob propagation metrics the most. The response of the blob to the individual interactions can, in many cases, be directly predicted from the source term equations. The case with all the sources included does, however, display a more complicated response to the source terms. It appears that the presence of all the sources affects the CoM motion more than just the sum of the individual sources, and, as seen in Fig. 1, some threshold is passed in which the asymmetrical perturbation is slowed down and no longer propagates as the front of the blob.

To get closer to the underlying mechanism that determines the motion of the blob with all interactions present, a scan in the individual moment sources is carried out in Sec. III B.

\section{B. Source term scan}

As a corroboration of the findings in Sec. III A, it is the interactions that introduce a density source or charge-exchange collisions that affect the blob motion the most; the results of a scan in the source terms (9)-(12) are presented. It is again constituted by a discrete sixpoint scan, which includes the case with all interactions, the case where no interactions are present, and the four cases where only one of the plasma source terms (9)-(12) is included and the others evaluated to zero in the nHESEL equations (1)-(4). To be clear, the difference of this scan from that in Sec. III A is that in the previous scan the interactions are included separately, but they can be sources or sinks to multiple fluid variables. In this scan, all interactions are included, but the resulting fluid variable source terms are included separately. Where the effect of, e.g., ionization of atoms is considered on its own in Sec. III A, the density/vorticity/pressure sources from ionization (and the other interactions) are included independently from one another in this scan.

In Figs. 6 and 7, the trajectories for "no interactions" and "all interactions" are identical to those in Figs. 2 and 3. It is observed that the CoM trajectories and velocities for the density and pressure sources alone are close to that for the blob without neutral interactions. The density and ion pressure sources accelerate slightly, whereas the electron pressure sink slows it down. The CoM trajectory and velocity for only the generalized vorticity source, on the other hand, are following those of the blob with all interactions included.

The analysis of the remaining metrics, i.e., the compactness and the thermal and kinetic energies of the blob, is similar and even more unambiguous for the source scan. In Fig. 8, the compactness rises with the density source, but is almost unaffected by the remaining source terms although the case with the vorticity source included also diverges more from the remaining cases without a direct density 

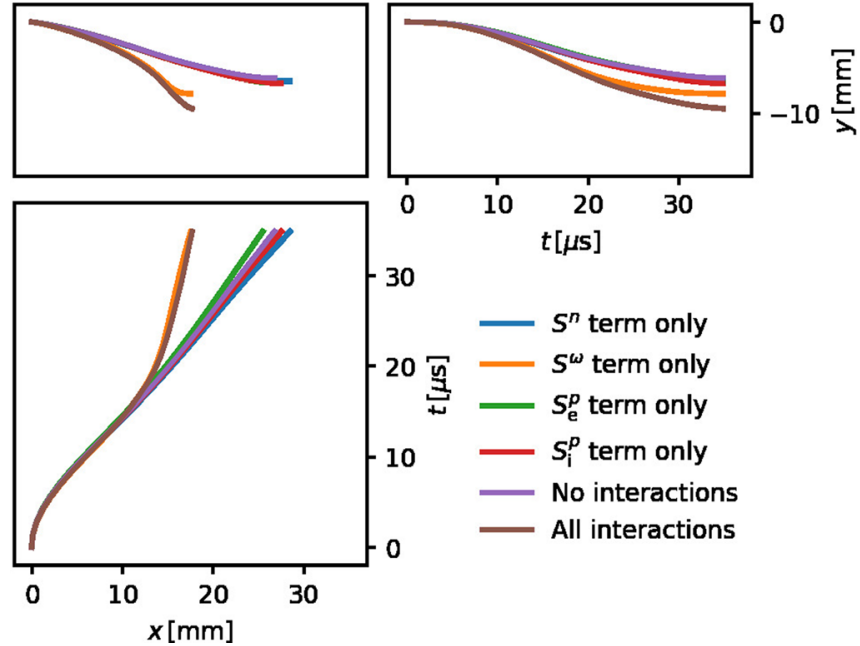

30

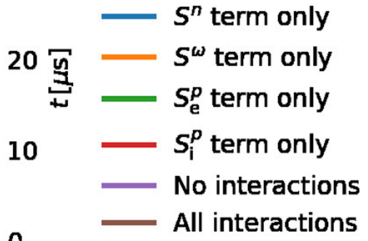

FIG. 6. Trajectory of the seeded blob center of mass position when, respectively, none, only one, and all of the sources in the plasma fields are accounted for. The axes are shared for the aligned frames as in Fig. 2. All neutral interactions are included in the source terms. It is observed that the scans divide in two fractions, which indicate that it is mainly the vorticity source that is responsible for the change in blob motion.

source. In Fig. 9, it is observed that the electron thermal energy evolution is defined by the electron pressure sink, the ion thermal energy is defined by the ion pressure source, and that the density source increases the blob kinetic energy, whereas it is decreased by the vorticity source. The pressure sources only affect the blob kinetic energy marginally.

In summary, it appears that, although the vorticity source plays a small role in most of the seeded blob evolution metrics, the blob CoM trajectory and the velocity are almost entirely defined by this source term. This is perhaps not surprising and agrees well with the
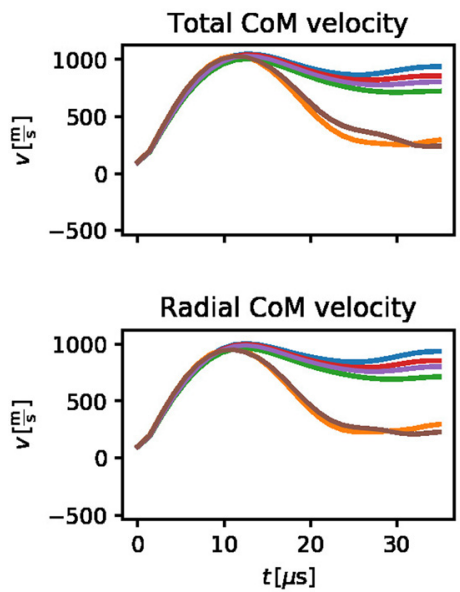

Poloidal CoM velocity

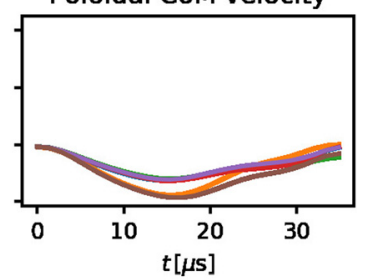

$S^{n}$ term only

$S^{\omega}$ term only

- $S_{\mathrm{e}}^{p}$ term only

$S_{i}^{p}$ term only

No interactions

All interactions
FIG. 7. Trajectory of the seeded blob center of mass position when, respectively, none, only one, and all of the sources in the plasma fields are accounted for. All neutral interactions are included in the source terms. As for Fig. 6, the vorticity source appears to be the main player in defining evolution of the velocity.
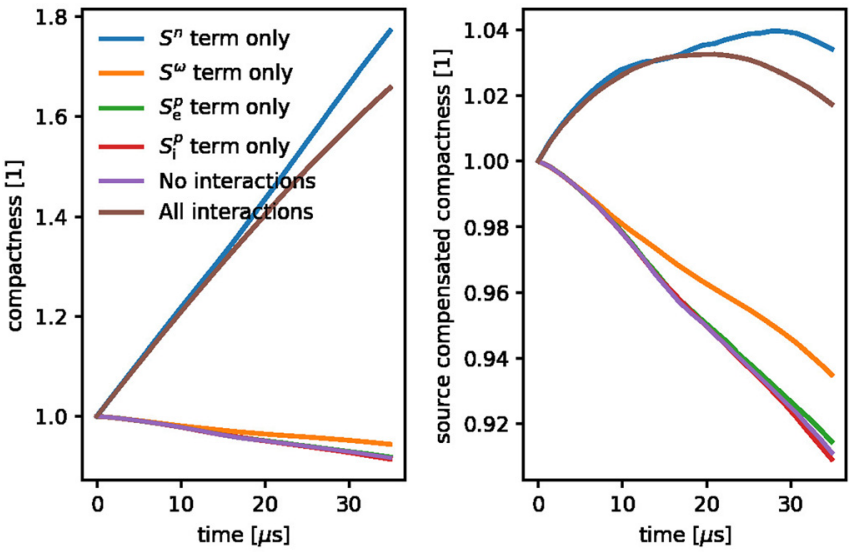

FIG. 8. Compactness and source compensated compactness of the perturbation in the source scan. The graphs for the no interactions and the pressure source scans are almost overlapping. Only the density source has a strong influence on the compactness. The presence of a vorticity source also increases the coherence of the filament.

observation in Sec. III A that the interactions that gave rise to a density source, together with charge-exchange, had the largest single impact on the blob CoM trajectory. It is exactly the density and chargeexchange source terms that appear in the vorticity source terms (10)-(13).

The discussion on the significant alteration of the blob dynamics is given in Sec. IV. Before Sec. IV, a scan in the blob parameters and the neutral background density is given in Sec. III C. This serves both
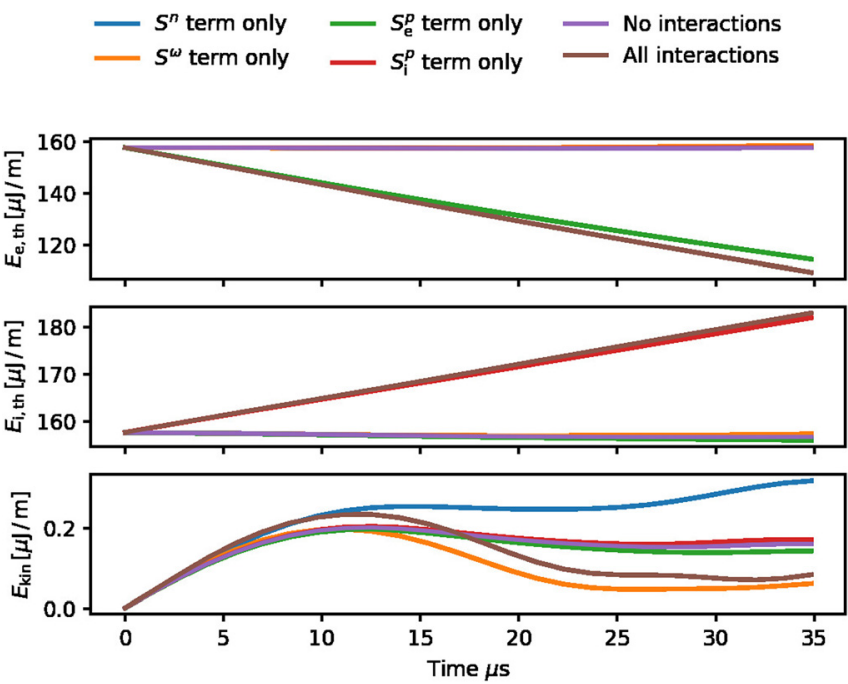

FIG. 9. Evolution of thermal electron (top frame) and ion (middle frame) energies, and the blob kinetic energy (bottom frame), under the influence of distinct sources from neutral interactions. The electron and ion pressure sources are the main drivers for the change in the corresponding thermal energies, but affect the kinetic energy weakly. The presence of a density source increases the kinetic energy, whereas the vorticity source decreases it compared to the case without source terms. 
as a check that the baseline parameters applied for the analysis are representative and thus insensitive to deviations around the chosen parameter value, and will also contribute to the discussion in Sec. IV.

\section{Reference and initialization parameter scan}

The center of mass position traces from a scan in the reference parameters $n_{0}, T_{\mathrm{e} 0}$, and the initial parameters $\sigma, n_{\mathrm{m}, \mathrm{a}}$ are shown in Fig. 10.

In Fig. 10(a), the change in the center of mass trace is consistent with the findings in Sec. III A, where it was found that it mattered only little whether the interaction introduced a density source or not, unless the source was sufficiently large (as for the case of all interactions), in which case the blob will slow down. Similarly in Fig. 10(a), the path changes only little when varying the reference density, up to the case for the largest reference density of $n_{0}=5 \times 10^{18} \mathrm{~m}^{-3}$, which also slowed down the blob in a similar manner.

In Figs. 10(c) and 10(d), a larger and systematical change of the center of mass trajectory with the change of the blob width $\sigma$ and reference temperature $T_{\mathrm{e} 0}$ is observed. The neutral interactions considered in Sec. III A have not pushed the blob into regimes as far away from the base case to find similarities as was done for the reference density scan. The systematical change of the blob transport with $\sigma$ and $T_{\mathrm{e} 0}$ is, however, expected from the previous studies ${ }^{4}$ and is explained well by the inertial scaling law, which is discussed in more detail in Sec. IV.

What stands out the most from the parameter scan is the behavior of the seeded blob with the highest neutral density background for which the center of mass trajectory is shown in Fig. 10(b). From all other cases, the trend is that the larger the density source, the more significant is the slow-down. A larger neutral density results in a larger
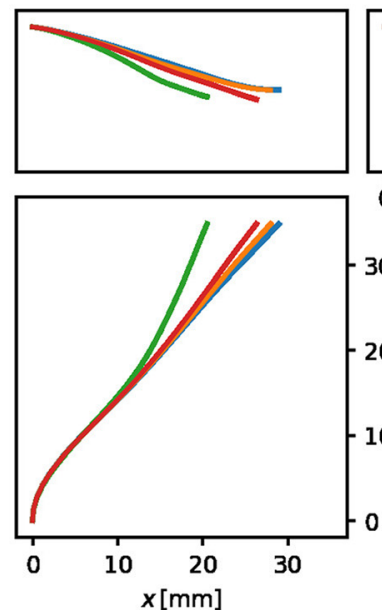

(a)
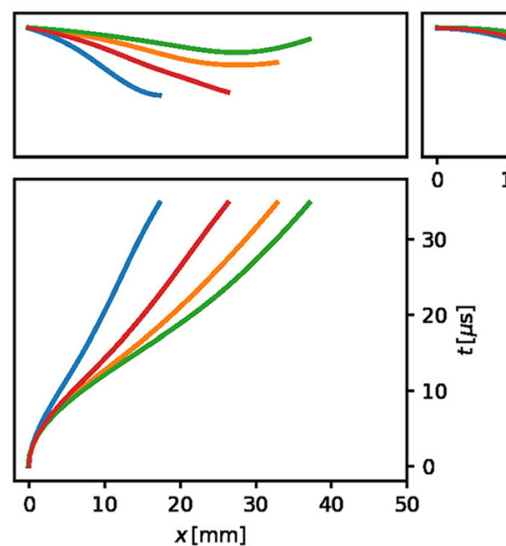

(c)
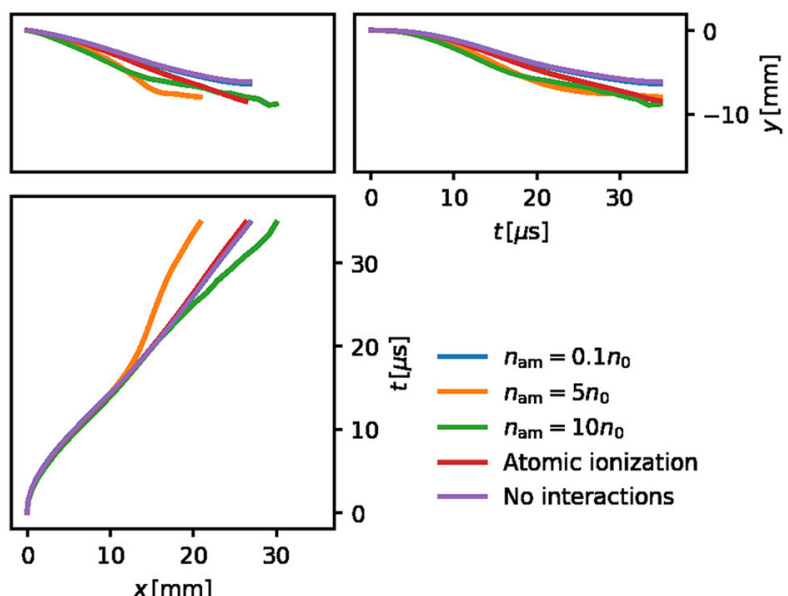

(b)
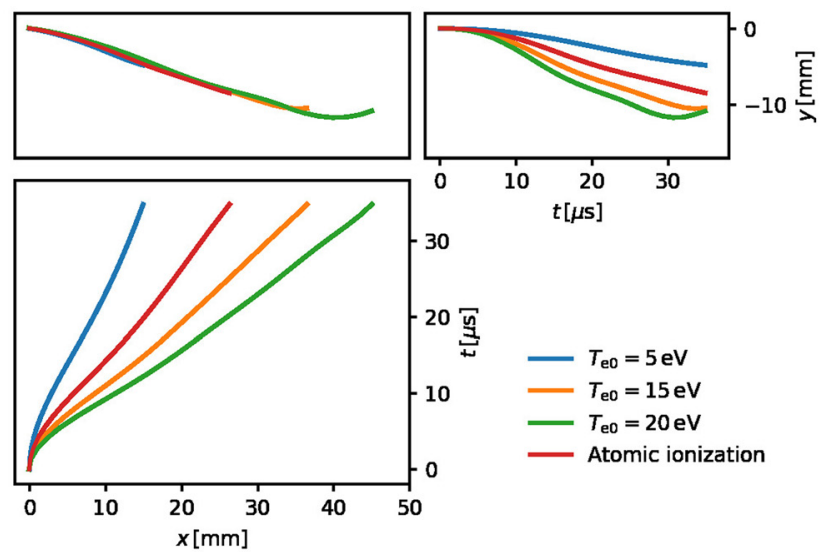

(d)

FIG. 10. Trajectories of the seeded blob center of mass position for a scan in the seeded blob density, temperature, and width parameters, and in the neutral density. The only neutral interaction included is atomic ionization. (a) $x_{\mathrm{COM}}$ for the scan in the electron density parameter $n_{0}$. The atomic ionization case has $n_{0}=1 \times 10^{18} \mathrm{~m}^{-3}$. (b) $x_{\mathrm{COM}}$ for the scan in the neutral atom and molecule density $n_{a m}$ in the multiple of the reference density $n_{0}=1 \times 10^{18} \mathrm{~m}^{-3}$. The atomic ionization case has $n_{\mathrm{am}}=n_{0}$. (c) $x_{\text {Com }}$ for the scan in the seeded blob width $\sigma$. The atomic ionization case has $\sigma=10 \rho_{\mathrm{s}}$. (d) $x_{\mathrm{Com}}$ for the scan in the electron temperature parameter $T_{\mathrm{e} 0 \text {. The atomic ionization case has }}$ $T_{\mathrm{e} 0}=10 \mathrm{eV}$. 
density source due to the linear dependency of both the ionization source terms (14) and (15), and a more pronounced slow-down would be expected for the higher neutral density case. For the simulation with the highest neutral density, however, it appears that some other mechanism accelerates the blob instead of slowing it down.

A possible explanation of the mechanisms that both slows down and speeds up the blobs at higher densities is discussed in Sec. IV.

\section{DISCUSSIONS}

In Sec. III A, it was observed that, if all source terms are active, the seeded blob slows down. The vorticity source term has been identified in Sec. III B as the main player, and it mediates the effect from the neutral interactions that introduce the density sources and momentum sinks, i.e., the ionization of molecules and atoms, and charge-exchange collisions between atoms and ions. Sources to the electron and ion pressures, on the other hand, only slightly alter the blob trajectory. The effect has been reproduced for other cases in Sec. III C, with the density source being stronger from either having a higher electron or a neutral density. It was, however, also observed that when the source is sufficiently strong, the blob speeds up instead of slowing down as seen from the CoM trajectory in Fig. 10(b).

To obtain some intuition on the fundamental mechanics of the source terms in the vorticity equation, a simpler model, similar to that of Refs. 4, 10, and 11, is considered. The model is a minimal example for interchange motion and describes the evolution of the vorticity $\omega=\nabla_{\perp}^{2} \phi / B$ for the electrostatic field $\phi$ and the magnetic field strength $B$. In the presence of a density source similar to (10), the vorticity equation reads

$$
\mathrm{D}_{t} \omega-\frac{2 c_{\mathrm{s}}^{2}}{R} \partial_{y} \log n=\nu_{\omega} \omega .
$$

The form of the source term is identical to that of (10) as the ion source drift in (13) is proportional to the ion $\mathbf{E} \times \mathbf{B}$ drift, and thus, the perpendicular gradient of the electrostatic potential, i.e., $\boldsymbol{u}_{\mathrm{i}}^{\mathrm{S}} \propto \boldsymbol{u}_{\mathrm{i}}$ $\propto \nabla_{\perp} \phi$, and the source term (10) are proportional to the vorticity as in (31). For a density source $S^{n}$, the relation to $\nu_{\omega}$ is $\nu_{\omega}=S^{n} / n$. For the case of a vanishing source (i.e., $\nu_{\omega}=0$ ), dimensional analysis recovers the inertial velocity scaling (see Refs. 4 and 11)

$$
v_{\text {inert }}=\sigma \gamma \frac{\delta n}{n},
$$

where $\sigma$ is the characteristic perpendicular blob length scale, and the characteristic interchange rate is given by

$$
\gamma=\left(\frac{2 c_{s}^{2}}{R \sigma}\right)^{\frac{1}{2}},
$$

where $c_{s}=\sqrt{T_{\mathrm{e}} / m_{\mathrm{i}}}$ is the ion sound speed and $R$ is the major radius of the machine. $\delta n$ is the perturbation to the background density $n$. For a finite density source, the reaction rate $\nu_{\omega}$ enters the blob velocity equation as

$$
v_{\text {blob }}=\frac{\sigma \gamma}{1+\nu_{\omega} / \gamma} \frac{\delta n}{n}=v_{\text {inert }} \frac{1}{1+\nu_{\omega} / \gamma} .
$$

From the dimensional analysis of the simple interchange model, one should expect the presence of a density source, such as the ionization of neutral particles, to slow down the blob. For the setup studies in this paper, the second factor in (34) is approximately

$$
\frac{1}{1+\nu_{\omega} / \gamma} \approx 0.98
$$

at the blob center at initialization for the case of atomic ionization. The slight decrease in the radial velocity observed in Fig. 3 for atomic ionization compared to the case without neutral interactions could be explained by the above analysis.

The behavior at stronger sources, in particular, for the neutral density scan in Sec. III C, does, however, suggest that other dynamics play a more dominant role. The above inertial scaling analysis is itself inadequate by only including the density source as an additional term in the vorticity equation (31); in Sec. II A, it has already been displayed that the ionization of atoms also affects the density and temperatures. Thus, for the inertial blob velocity, one would not only expect a decrease due to the factor in (34), but also a decrease of the inertial blob velocity caused by the sink in the electron temperature (affecting $c_{s}$ and thus $\gamma$ ) as well as an increase due to the increased density (affecting $\delta n$ ). The conclusions drawn by the analysis in Sec. III B are, however, that the changes to the plasma pressures do not affect the blob significantly and that the main driver for the change to the blob dynamics is indeed the vorticity source.

A more satisfying explanation for the failure of the simplified analysis is the lack of the finite Larmor radius (FLR) effects in (31), which are present in the nHESEL vorticity equation (2). The FLR effects result from the large ratio of ion to electron masses and manifest when ions are described with a finite temperature. In Ref. 7, the FLR terms are derived for a two-moment gyrofluid model, and the effects on seeded blobs are described in detail. It is found that FLR effects break the up-down symmetry, which causes the blob to move in the $\mathbf{B} \times \nabla \mathbf{B}$ direction and increase the coherence of the blob. It should be noted that the poloidal dynamics are also partly determined by the initial conditions for the electric potential. ${ }^{18}$ The FLR effects manifest in the vorticity equation and effectively generate a sheared flow surrounding the blob, which reduces mixing, stretching, and generation of small scale density fluctuations. The sheared flow is associated with an advection of vorticity along the density contour lines, and the effect is visible in the top row of frames in Fig. 11. It is also noted in Ref. 7 that the dynamics in the later phase are strongly non-linear, which complicates the analysis of the late-stage blob evolution.

Figure 11 shows the evolution of the structure of the generalized vorticity for three of the cases examined in Sec. III C, where only the atomic ionization interaction is active and the neutral density is increased from $n_{0}$ (top frames) through $5 n_{0}$ (middle frames) to $10 n_{0}$ (bottom frames) and is thus effectively a scan in $\nu_{\omega}$ at the ratios of 1 , 5 , and 10 to the base case. The stronger vorticity source at the blob center causes the vorticity layer surrounding the blob, as seen in the top frame, to increase, which slows down the blob as depicted in the middle frames, before shattering the layered structure toward the end of the simulation. The increase in the amplitude of the surrounding vorticity layer slows the blob down, as observed in Sec. III C. It is also this effect that is causing the slow-down of the blob with all interactions present in Sec. III A and only the vorticity source active in Sec. III B. For a stronger source, the loss of the vorticity structure happens at an even earlier stage before the blob slows down and the lack of structure apparently accelerates the blob slightly compared to the base 

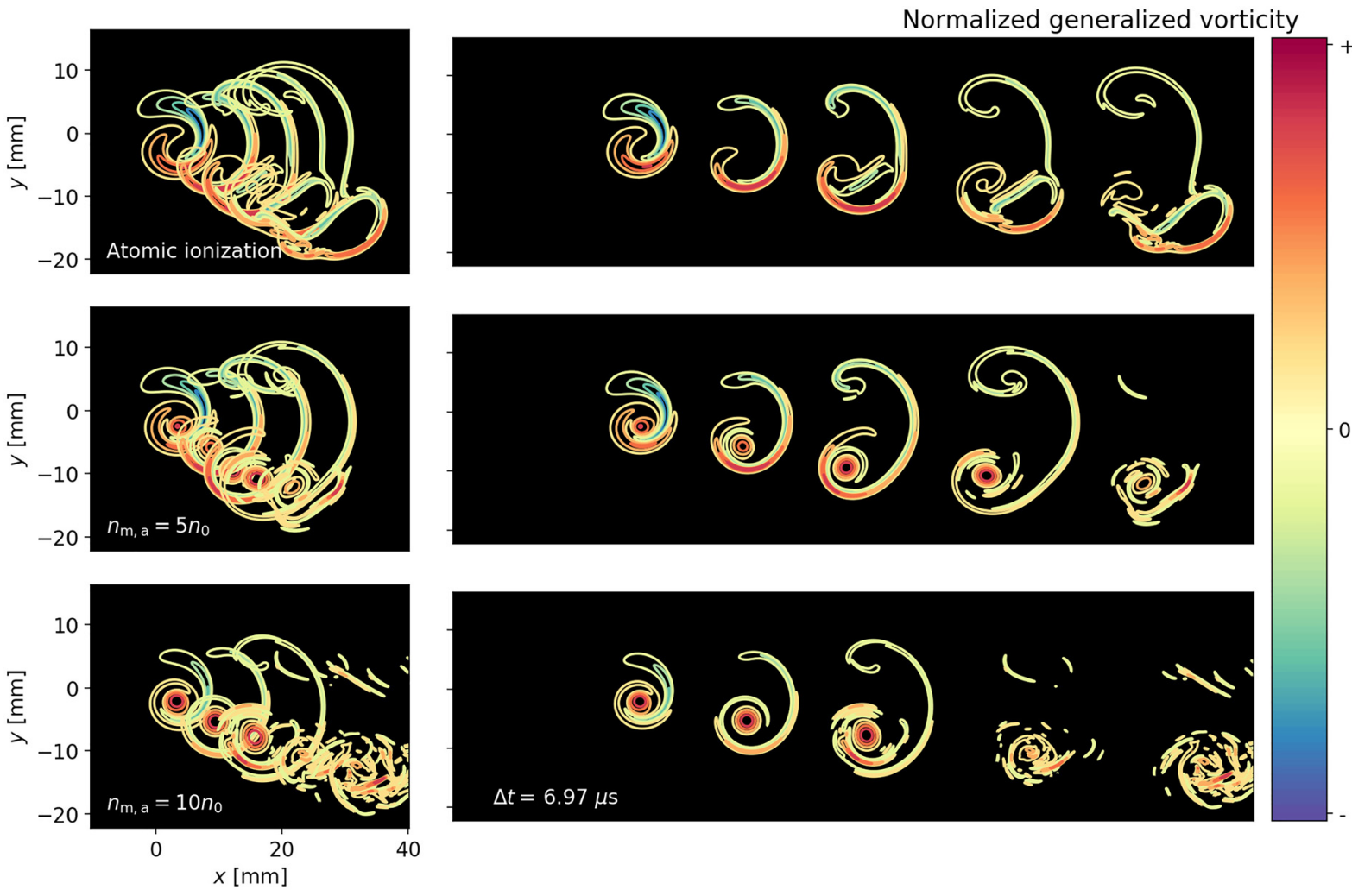

FIG. 11. Evolution of the structure of the generalized vorticity for a seeded blob with only the atomic ionization source (15) active at the neutral density levels of $n_{\mathrm{m}, \mathrm{a}}=n_{0}, 5 n_{0}, 10 n_{0}$. Due to the strong increase in the absolute value of the vorticity, the colorbar is renormalized at every time step and does only carry qualitative information.

case where the layer of the sheared flow around the perturbation is intact. Whether the blob slows down or speeds up in the later phase of its evolution thus depends on the strength of the source compared to the interchange time, i.e., the dimensionless parameter $\nu_{\omega} / \gamma$, which also appears in (34) in the inertial scaling analysis.

With a better understanding of how strong sources from neutral interactions influence the blob dynamics, the discussion is briefly turned to the topic of whether such changes influence the SOL filament dynamics.

An observed phenomenon of particular relevance is the flattening of the density profile and the formation of a "shoulder" in the far SOL region. ${ }^{19,20}$ Although the shoulder formation is readily produced in experiments, see, e.g., Refs. 21 and 22, the underlying mechanisms are still debated.

There is a general consensus that the density flattens as a result of changes to the filament dynamics. It has been found that the increased flattening of the far SOL density is correlated with an increase in the blob size and a small variation of the radial velocity both experimentally ${ }^{21,23,24}$ and in SOL turbulence simulations. ${ }^{25}$ One hypothesis for the change of filament dynamics is the increment of divertor collisionality $\left(\Lambda_{\text {div }}\right)$, see, e.g., Ref. 21 , and thus the reduction of parallel conductivity in the filaments. This changes the SOL from a sheath-limited to an inertial regime, which again changes the blob dynamics. It has, however, also been found ${ }^{24}$ that increasing $\Lambda_{\text {div }}$ is by itself not necessarily sufficient for a density shoulder to form, and a feedback loop from the ionization of neutrals in the far SOL region is proposed in Ref. 8. Experiments have also revealed a change in the near SOL filament ion temperature, which decreases significantly during the transition from a low to high divertor collisionality. ${ }^{26}$

The results presented in this paper are interesting in the framework of shoulder formation; the high plasma density required for the shoulder to form is often obtained from gas puffing, which causes a high SOL neutral density and thus a strong increase in $\nu_{\omega}$. The effect of the considered interactions on the filaments is consistent with the observed increase in the filament size (from the density sources) and the change of the filament cross-field velocity (from the vorticity sources). The simulations show an increase in the ion temperature when more neutral interactions are included. This effect is, however, mostly due to an artifact that arises from describing the ions as a single fluid, and not directly comparable to the results of Ref. 26, where an ion temperature decrease is observed for an increased divertor collisionality. The seeded blob study is, however, not sufficient to determine if inelastic neutral sources play an important role in the formation of density shoulders. It is interesting, however, that the results point toward a dual role of the ionization of neutrals as being not only a density source but together with charge-exchange collisions to modify the vorticity and thus the blob dynamics. The next step in this direction is a similar investigation for a full edge/SOL turbulence simulation, which is planned for the near future. 


\section{CONCLUSIONS}

Seeded blob simulations are a common tool for studying isolated effects on the SOL cross-field filamentary transport. In this paper, the effects of inelastic plasma-neutral interactions that are common in the SOL region, including the ionization of molecules and atoms and charge-exchange collisions between ions and atoms, have been investigated. The conclusions are found on the results from a series of discrete scans.

The changes in inertial blob dynamics from including the interactions one by one in Sec. III A reveal that the interactions that are sources or sinks to the zeroth (density) and first (momentum) order plasma fluid variables influence the blob dynamics much more than sources to the pressures. This result is supported by a scan where all interactions are present, but the corresponding source terms are included independently one by one in Sec. III B. Sources and sinks to the pressure equations, on the other hand, only affect the blob dynamics marginally.

In particular, the density sources influence the blob compactness and the so-called source compensated compactness. The compactness of the blob increases strongly in the presence of a density source. The source compensated compactness also shows an initial increase, which is, however, not sustained. The terminal decline of the source compensated compactness is slower than for the cases without a density source. The evolution of the source compensated compactness thus suggests that a density source increases the blob lifetime by delaying the dispersion and lowering the dispersion rate.

The density and momentum sources and sinks constitute a drift term, which influences the vorticity directly by introducing a vorticity source from the divergence of this drift velocity, whereas the higher moment source terms do not enter the vorticity equation directly. The change in the vorticity structure generally causes the blob center of mass radial velocity to decrease for a stronger source. The opposite effect, i.e., a radial acceleration of the center of mass, is, however, observed for the case of a strong density source. The interplay between the vorticity source and the finite Larmor radius effects, which result from the disparity in the electron ion masses and also enter the vorticity equation at a finite ion temperature, thus affects the blob dynamics in a non-linear manner. The significance of this is, to some extent, determined by the ratio of the source rate to the interchange time.

It is discussed whether inelastic interactions with neutrals may play a role in the formation of SOL density shoulders. It is argued that the density source may contribute to the formation of a density shoulder not only by increasing the density locally but also, together with momentum sinks from, e.g., charge-exchange collisions and neutral friction, by changing the blob dynamics through the vorticity. The results from this paper thus indicate that the effects of the sources on the filaments are consistent with those observed experimentally, but further investigations are required to draw any conclusive connections between inelastic sources from plasma-neutral interactions and the shoulder formation. Such investigations are underway where the local effect of neutral self-consistent interactions with a turbulent SOL plasma is investigated.

\section{ACKNOWLEDGMENTS}

This work has been carried out within the framework of the EUROfusion Consortium and has received funding from the
Euratom research and training programs 2014-2018 and 2019-2020 under Grant Agreement No. 633053. The views and opinions expressed herein do not necessarily reflect those of the European Commission.

\section{REFERENCES}

${ }^{\text {1} S . ~ J . ~ Z w e b e n, ~ J . ~ A . ~ B o e d o, ~ O . ~ G r u l k e, ~ C . ~ H i d a l g o, ~ B . ~ L a B o m b a r d, ~ R . ~ J . ~ M a q u e d a, ~}$ P. Scarin, and J. L. Terry, Plasma Phys. Controlled Fusion 49, S1 (2007).

${ }^{2}$ S. I. Krasheninnikov, Phys. Lett. A 283(5-6), 368-370 (2001).

${ }^{3}$ D. A. D’Ippolito, J. R. Myra, and S. J. Zweben, Phys. Plasmas 18, 060501 (2011).

${ }^{4}$ O. E. Garcia, N. H. Bian, V. Naulin, A. H. Nielsen, and J. Juul Rasmussen, Phys. Plasmas 12, 090701 (2005).

${ }^{5}$ O. E. Garcia, N. H. Bian, and W. Fundamenski, Phys. Plasmas 13, 082309 (2006).

${ }^{6}$ J. Olsen, J. Madsen, A. H. Nielsen, J. Juul Rasmussen, and V. Naulin, Plasma Phys. Controlled Fusion 58, 044011 (2016).

${ }^{7}$ J. Madsen, O. E. Garcia, J. S. Larsen, V. Naulin, A. H. Nielsen, and J. Juul Rasmussen, Phys. Plasmas 18, 112504 (2011).

${ }^{8}$ D. Carralero, M. Siccinio, M. Komm, S. A. Artene, F. A. D’Isa, J. Adamek, L. Aho-Mantila, G. Birkenmeier, M. Brix, G. Fuchert et al., Nucl. Fusion 57, 056044 (2017).

${ }^{9}$ A. Wynn, B. Lipschultz, I. Cziegler, J. Harrison, A. Jaervinen, G. F. Matthews, J. Schmitz, B. Tal, M. Brix, C. Guillemaut et al., Nucl. Fusion 58, 056001 (2018).

${ }^{10}$ R. Kube and O. E. Garcia, Phys. Plasmas 19, 042305 (2012).

${ }^{11}$ C. Theiler, I. Furno, P. Ricci, A. Fasoli, B. Labit, S. H. Müller, and G. Plyushchev, Phys. Rev. Lett. 103, 065001 (2009).

${ }^{12}$ A. S. Thrysøe, M. Løiten, J. Madsen, V. Naulin, A. H. Nielsen, and J. Juul Rasmussen, Phys. Plasmas 25, 032307 (2018).

${ }^{13}$ A. H. Nielsen, G. S. Xu, J. Madsen, V. Naulin, J. Juul Rasmussen, and B. N. Wan, Phys. Lett. A 379, 3097-3101 (2015).

${ }^{14}$ J. Juul Rasmussen1, A. H. Nielsen, J. Madsen, V. Naulin, and G. S. Xu, Plasma Phys. Controlled Fusion 58, 014031 (2016).

${ }^{15}$ J. Madsen, V. Naulin, A. H. Nielsen, and J. J. Rasmussen, Phys. Plasmas 23, 032306 (2016).

${ }^{16}$ S. I. Braginskii, Rev. Plasma Phys. 1, 205 (1965), available at https://ui.adsabs. harvard.edu/abs/1965RvPP...1..205B/abstract.

${ }^{17}$ B. D. Dudson, M. V. Umansky, X. Q. Xu, P. B. Snyder, and H. R. Wilson, Comput. Phys. Commun. 180, 1467 (2009).

${ }^{18} \mathrm{M}$. Held, "Full-F gyro-fluid modelling of the tokamak edge and scrape-off layer," Ph.D. thesis (University of Innsbruck, 2016).

${ }^{19}$ B. LaBombard, M. V. Umansky, R. L. Boivin, J. A. Goetz, J. Hughes, B. Lipschultz, D. Mossessian, C. S. Pitcher, J. L. Terry, and Alcator Group, Nucl. Fusion 40, 2041 (2000).

${ }^{20}$ D. L. Rudakov, J. A. Boedo, R. A. Moyer, P. C. Stangeby, J. G. Watkins, D. G. Whyte, L. Zeng, N. H. Brooks, R. P. Doerner, T. E. Evans et al., Nucl. Fusion 45, 1589 (2005).

${ }^{21}$ D. Carralero, H. W. Müller, M. Groth, M. Komm, J. Adamek, G. Birkenmeier, M. Brix, F. Janky, P. Hacek, S. Marsen et al., J. Nucl. Mater. 463, 123-127 (2015).

${ }^{22}$ N. Vianello, D. Carralero, C. K. Tsui, V. Naulin, M. Agostini, I. Cziegler, B. Labit, C. Theiler, E. Wolfrum, D. Aguiam et al., Nucl. Fusion 60, 016001 (2020).

${ }^{23}$ D. Carralero, P. Manz, L. Aho-Mantila, G. Birkenmeier, M. Brix, M. Groth, H. W. Müller, U. Stroth, N. Vianello, E. Wolfrum et al., Phys. Rev. Lett. 115, 215002 (2015).

${ }^{24}$ N. Vianello, C. Tsui, C. Theiler, S. Allan, J. Boedo, B. Labit, H. Reimerdes, K. Verhaegh, W. A. J. Vijvers, N. Walkden et al., Nucl. Fusion 57, 116014 (2017).

${ }^{25}$ A. H. Nielsen, J. Juul Rasmussen, J. Madsen, G. S. Xu, V. Naulin, J. M. B. Olsen, M. Løiten, S. K. Hansen, N. Yan, L. Tophøj et al., Plasma Phys. Controlled Fusion 59, 025012 (2017).

${ }^{26}$ D. Carralero, S. Artene, M. Bernert, G. Birkenmeier, M. Faitsch, P. Manz, P. de Marne, U. Stroth, M. Wischmeier, E. Wolfrum et al., Nucl. Fusion 58, 096015 (2018). 